\title{
The association between critical life events, sociodemographic data and physical activity in the development of myocardial infarction in smokers and ex-smokers
}

\author{
Anna Schmidt ${ }^{1 \#}$, Elke Driller ${ }^{1}$, Melanie Neumann ${ }^{2}$, Julia Jung ${ }^{1}$, Andrea Staratschek-Jox ${ }^{3}$, \\ Christian Schneider ${ }^{4}$, Jürgen Wolf ${ }^{5}$, Holger Pfaff ${ }^{1}$ \\ ${ }^{1}$ Institute for Medical Sociology, Health Services Research and Rehabilitation Science (IMVR), Faculty of Human Science and Fac- \\ ulty of Medicine, University of Cologne, Cologne, Germany; ${ }^{\#}$ Corresponding Author: anna.schmidt@uk-koeln.de \\ ${ }^{2}$ Gerhard Kienle Institute for Medical Theory, Integrative and Anthroposophic Medicine, Integrated Curriculum for Anthroposophic \\ Medicine (ICURAM), Medical Department of the Private, University of Witten/Herdecke, Witten, Germany \\ ${ }^{3}$ LIMES (Life and Medical Sciences Bonn), Genomics and Immunoregulation, University of Bonn, Bonn, Germany \\ ${ }^{4}$ Department III for Internal Medicine, University Hospital of Cologne, Cologne, Germany \\ ${ }^{5}$ First Department of Internal Medicine, Molecular Tumour Biology and Tumour Immunology \& Centre for Integrated Oncology \\ (CIO), University Hospital of Cologne, Cologne, Germany
}

Received 9 March 2012; revised 24 April 2012; accepted 18 May 2012

\begin{abstract}
Background: Psychosocial factors attract interest in investigating the occurrence of cardiovascular disease. Design this study aimed to examine the impact of critical life events on the development of myocardial infarction in smokers and ex-smokers. We hypothesized that critical life events increase the risk of the disease. Methods: Data were taken from the Cologne Smoking Study (CoSmoS), a retrospective multicentre case-control study that examines which psychosocial factors may lead to a higher risk for smokers and ex-smokers of suffering from a myocardial infarction. Our sample consisted of $n=278$ myocardial infarction participants and control participants. Both groups had a history of smoking. Logistic regression was used in the analysis. Results: The study results of the smoking and ex-smoking participants showed that sociodemographic data like gender and age have an effect on the development of myocardial infarction. Physical activity seems to offer protection aganist myocardial infarction. Final, the unexpected result that the experience of at least one critical life event seems to have a positive effect on health and so lowers the risk of myocardial infarction. Conclusions: Sociodemographic data and physical activity have an effect on the development of myocardial infarcttion. Par-
\end{abstract}

*Disclosure: No conflict of interest. ticipants with experience of critical life events appear to be strengthened after the events and possess adequate resources to protect their health.

Keywords: Life Events; Physical Activity; Myocardial Infarction; Smokers

\section{INTRODUCTION}

Ischaemic heart disease is the leading cause of death worldwide [1]. In $2008,12.8 \%$ of all deaths were the result of ischemic heart disease [2,3]. One of the risk factors for myocardial infarction is smoking [4]. Worldwide, approximately 1.3 billion people - currently smoke cigarettes or use other tobacco products [5]. Given, however, that not every smoker will necessarily suffer from a myocardial infarction, more attention has been paid to various psychosocial conditions as risk factors for myocardial infarction [6].

\subsection{Critical Life Events}

Generally speaking, critical life events are situations that create rifts and discontinuities in life circumstances and therefore challenge an individual to adapt [7]. These events are considered extraordinary types of experiences because they differ from "typical" age-graded life transitions (the first day of school, puberty, etc.), making them non-normative in nature, and because of their uniqueness. An event is critical when life after the event is no longer what it was before. 
The main instrument used for measuring critical life events is Holmes and Rahe's Social Readjustment Scale [8]. The scale assesses everyday stressors (e.g. unemployment, illness, social conflict, accident, death of a loved one) that negatively impact on the development of a disease [9]. The sum of all critical life events occurring during a given period of time is seen as a measure of the stress experienced during that time [10]. In the present study, critical life events constituted preconditions, which, depending on an individual's ability to cope, can have more or less profound effects on a number of important areas of life, and as a result, on the individual's health [7].

\subsection{Empirical Findings}

Rafanelli et al. [11] investigated the relationship between stressful life events (e.g. employment, financial status, family and social problems) and acute myocardial infarction as part of their study. Participants in their study who had suffered a myocardial infarction reported significantly more stressful life events than the healthy participants. The researchers in the team led by Kornerup [12] found that major live events increase the risk of stroke but not of myocardial infarction. In a study by Hollis et al. [13], a total of 31 items were used to assess life events including work, marriage, legal or financial difficulties, accidents and crime. The results of this six-year follow-up study indicated no association between annual accumulations of life events and cardiovascular outcomes such as myocardial infarction and angina pectoris.

The empicical findings showed that we found several studies investigating the relationship between critical life events and the onset of illness [14]. We found no study, however, which exclusively investigated myocardial infarction patients with a history of smoking. At this point the present study begins.

\subsection{Aim of the Study}

Our explorative study aimed to determine whether there is an association between stressful life events and the onset of myocardial infarction for people with a history of smoking. Using the design of the Cologne Smoking Study (CoSmoS), we were able to examine the hypothesis that smokers and ex-smokers who experience critical life events are at a higher risk of myocardial infarction.

\section{METHODS}

\subsection{Study Design and Participants}

Data for the study (survey period: 2004-07) were taken from CoSmoS, a multicentre case-control study that ex- amines which genetic and/or psychosocial factors put smokers and ex-smokers at higher risk of suffering a myocardial infarction, developing lung cancer and/or becoming addicted to nicotine. The study was approved by the Ethics Committee of the University Hospital of Cologne (UHC). Two case-study groups and one hospital-based control group were recruited for the study. To be included, participants in all three groups had to be of European descent, reside in or around the city of Cologne and be born between 1930 and 1970. The main phase of the study lasted two years (for the study design see also [15]).

Of the $\mathrm{n}=524$ participants included in CoSmoS, 458 $(87.4 \%)$ were smokers or ex-smokers and $66(12.6 \%)$ were non-smokers. 180 lung cancer patients and 170 myocardial infarction patients (acute and/or with a history of myocardial infarction) were recruited from Lung Clinic Merheim and the UHC. 174 control group patients, who had not been diagnosed with either condition and who did not have an admission diagnosis of a cancer and/or nicotine-related disease, were selected from the Orthopaedics and Dermatology departments at the UHC. The choice for controls was group matching by means of sex, age, residence and smoking behavior.

Potential participants were approached by a study nurse on the wards of the different departments. Patients who met the inclusion criteria were asked to sign a consent form before participating in the study. Participants were surveyed in hospital through face-to-face interviews, with each interview lasting an average of 45 to 60 minutes.

For the present analysis, only data from smokers and ex-smokers with myocardial infarction and from the control group were used (see Figure 1). Both groups were asked the same questions.

\subsection{Measures}

Study participants were surveyed about critical life events by means of a questionnaire based on Holmes and Rahe's Social Readjustment Scale [16]. Among the types of major life events, or macrostressors, included in the survey were illness, impairment, accident, death, unemployment, circumstances at work, financial worries, conflict with a loved one, change of residence, separation from a spouse or partner, increase in family size and pregnancy. A distinction was made between events pertaining to the individual and to the individual's loved ones. When answering, participants were asked to recall events that had occurred during the course of the year prior to the first diagnosis of their most recent myocardial infarction (case-study group) or their current disease or condition (control group). Response options for the occurrence of each event were "yes" and "no". 


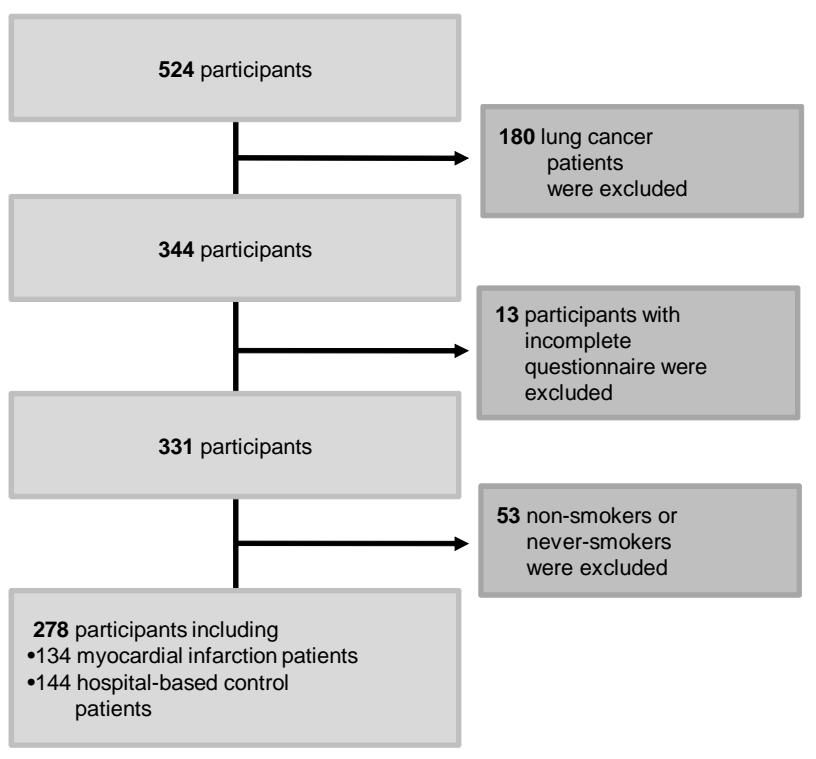

Figure 1. Flowchart of the sampling procedure.

For the analysis we chose the events "severe personal illness", "personal accident" and "increased personal demands at work", which were named frequently.

Sociodemographic and other data (e.g. gender, age, physical activity, smoking status) taken from the comprehensive questionnaire of CoSmoS were included in the analysis as additional control and moderator variables.

Data on gender were obtained, as usual, by asking participants whether they were "male" or "female". The age variable was assessed by asking patients when they were born and then categorizing them into ten-year age groups $(1=35-44 ; 2=45-54 ; 3=55-64 ; 4=65-76)$ for the analysis.

Patients' religion was assessed by asking them whether they adhered to any religion (e.g. Christianity, Sikhism, Islam, Judaism) or no religion. For analytical purposes, the religious categories were dichotomized into "religious" and "not religious".

To measure physical activity, patients were asked about their activity during the year prior to the first diagnosis of their current condition. Responses were coded as 0 (not physically active during the week) and 1 (physically active at least one hour per week).

The categorical variable "pack years", a measure of the cumulative number of cigarettes smoked by an individual, was calculated by multiplying the number of cigarettes smoked per day by the number of years the person had smoked divided by 20 [17]. Smoking exposure was defined by pack years: none, 15 or fewer, more than 15 [18]. Pipe and cigar smoking were not considered in this study.

For smoking status, a distinction was made between current smokers and ex-smokers. Patients were asked at what age they had stopped smoking. Patients who had given up smoking at least five years prior to the study were considered ex-smokers [4].

\subsection{Data Analysis}

Figure 1 illustrates the sampling procedure for the statistical analysis of the association between critical life events and myocardial infarction.

A two-step analysis was conducted. First, Spearman and chi-square tests of association were performed on the study variables to determine whether there was a statistically significant difference in the means of two independent samples. Next, we tested our hypothesis using a stepwise logistic regression model because the logistic function was needed to estimate the probability that study participants would belong to one of the binary dependent variable categories (coded 0 for control patients and 1 for myocardial infarction patients). P-values $\leq 0.05$ were considered to be statistically significant.

\section{RESULTS}

\subsection{Descriptive Findings}

Our sample consisted of $n=278$ participants, of whom $n=134$ were myocardial infarction patients and $n=144$ were control group patients. The distributions of life events and the other independent variables are shown in Table 1.

\subsection{Bivariate Analysis}

The results of the Spearman and chi-square tests yielded no significant correlations between the independent variables (results not shown here). Similarly, none of the variables under investigation demonstrated intercorrelations $>0.80$, which indicated that there was no multicollinearity [19].

\subsection{Multivariate Analysis}

The results of the stepwise logistic regression are shown in Table 2.

In the following, we report the results from Model 2. (Please see Table 2 for the results from Model 1) The experience of at least one of the critical life events measured (severe personal illness, a personal accident, or increased personal demands at work) reduced the participant's likelihood of suffering a myocardial infarction (adjusted OR $=0.99^{*}$ ).

The result shows also that being female (adjusted OR $=0.29^{* * *}$ ) and being younger than 44 (adjusted OR $=$ $4.90^{* *}-5.42^{* *}$ ) have a protective effect on the development of myocardial infarction. Participants with physical activity have a lower risk of a myocardial infarction 
Table 1. The characteristics of the study sample $(\operatorname{CoSmoS} n=278)$.

\begin{tabular}{|c|c|c|c|c|c|c|}
\hline \multirow{2}{*}{ Variable } & \multirow{2}{*}{ Coding } & \multirow{2}{*}{$\begin{array}{c}\text { Missing values } \\
\mathrm{n}\end{array}$} & \multicolumn{2}{|c|}{ Myocardial infarction patients } & \multicolumn{2}{|c|}{ Control patients } \\
\hline & & & $\mathrm{n}$ & $\%$ & $\mathrm{n}$ & $\%$ \\
\hline \multirow{2}{*}{ Gender } & male & 0 & 114 & 41.0 & 89 & 32.0 \\
\hline & female & 0 & 20 & 7.2 & 55 & 19.8 \\
\hline \multirow{4}{*}{ Age } & $35-44$ & \multirow{4}{*}{1} & 5 & 1.8 & 22 & 7.9 \\
\hline & $45-54$ & & 37 & 13.4 & 31 & 11.2 \\
\hline & $55-64$ & & 46 & 16.6 & 52 & 18.8 \\
\hline & $65-76$ & & 46 & 16.6 & 38 & 13.7 \\
\hline \multirow{2}{*}{ Religion } & religious & 0 & 91 & 67.9 & 107 & 74.3 \\
\hline & not religious & 0 & 43 & 32.1 & 37 & 25.7 \\
\hline \multirow{2}{*}{ Physical activity } & physically active & 0 & 42 & 15.1 & 72 & 25.9 \\
\hline & not physically active & 0 & 92 & 33.1 & 72 & 25.9 \\
\hline \multirow{2}{*}{ Pack years } & $\leq 15$ pack years & \multirow{2}{*}{19} & 60 & 23.2 & 77 & 29.7 \\
\hline & $>15$ pack years & & 66 & 25.5 & 56 & 21.6 \\
\hline \multirow{2}{*}{ Smoking status } & current smoker & 0 & 81 & 29.1 & 75 & 27.0 \\
\hline & ex-smoker & 0 & 53 & 19.1 & 69 & 24.8 \\
\hline \multirow{4}{*}{ Life events } & 0 & 0 & 101 & 75.4 & 91 & 63.2 \\
\hline & 1 & 0 & 26 & 19.4 & 29 & 20.1 \\
\hline & 2 & 0 & 7 & 5.2 & 22 & 15.3 \\
\hline & 3 & 0 & 0 & 0 & 2 & 1.4 \\
\hline
\end{tabular}

Table 2. The results of a stepwise logistic regression $(\operatorname{CoSmoS} n=278)$.

\begin{tabular}{|c|c|c|c|c|c|c|c|c|}
\hline \multirow{2}{*}{ Independent variable } & \multicolumn{4}{|c|}{ Step 1} & \multicolumn{4}{|c|}{ Step 2} \\
\hline & Beta & SE & OR & CI & Beta & SE & Adjusted OR & CI \\
\hline Gender $\left(\mathrm{male}^{\#}\right)$ & -1.271 & 0.320 & $0.28^{* * *}$ & & -1.238 & 0.323 & $0.29^{* * *}$ & $0.154-0.546$ \\
\hline \multicolumn{9}{|l|}{ Age } \\
\hline \multicolumn{9}{|l|}{$35-44^{\#}$} \\
\hline $45-55$ & 1.687 & 0.581 & $5.40^{* *}$ & & 1.587 & 0.582 & $4.90^{* *}$ & $1.562-15.306$ \\
\hline $55-64$ & 1.431 & 0.573 & $4.18^{* *}$ & & 1.381 & 0.572 & $3.98^{*}$ & $1.296-12.217$ \\
\hline $65-76$ & 1.721 & 0.595 & $5.59^{* *}$ & & 1.689 & 0.593 & $5.42^{* *}$ & $1.693-17.324$ \\
\hline Religion (not religious ${ }^{\#}$ ) & -0.122 & 0.297 & 0.89 & & -0.154 & 0.300 & 0.86 & $0.477-1.543$ \\
\hline Physical activity (not active ${ }^{\#}$ ) & -0.627 & 0.272 & $0.53^{*}$ & & -0.625 & 0.275 & $0.54^{*}$ & $0.312-0.917$ \\
\hline Pack years $\left(\leq 15^{\sharp}\right)$ & -0.003 & 0.002 & 1.00 & & -0.004 & 0.003 & 1.00 & $0.312-0.917$ \\
\hline Smoking status (ex-smoker $\left.{ }^{\#}\right)$ & 0.270 & 0.313 & 0.71 & & 0.171 & 0.318 & 1.19 & $0.635-2.214$ \\
\hline Life events $\left(\right.$ none $\left.^{\#}\right)$ & & & & & -0.011 & 0.005 & $0.99^{*}$ & $0.978-0.999$ \\
\hline Cox and Snell pseudo- $\mathrm{R}^{2}$ & 0.14 & & & & 0.16 & & & \\
\hline Nagelkerke pseudo- $\mathrm{R}^{2}$ & 0.20 & & & & 0.21 & & & \\
\hline McFadden pseudo- $\mathrm{R}^{2}$ & 0.11 & & & & 0.13 & & & \\
\hline
\end{tabular}

Note: ${ }^{\#}=$ reference response, Beta $=$ regression coefficient, $\mathrm{SE}=$ standard error, $\mathrm{OR}=$ odds ratio, $\mathrm{CI}=$ confidence interval, ${ }^{*} \mathrm{p} \leq 0.05,{ }^{* *} \mathrm{p} \leq 0.01,{ }^{* * *} \mathrm{p} \leq 0.001$. 
(adjusted OR $=0.54^{*}$ ). The results of the other variables whose odds ratios are not statistically signifycant can be found in Table 2. In Model 2 the Nagelkerke pseudo- $\mathrm{R}^{2}$ was $21 \%$ (for the other coefficients, see Table 2). The specificity of the second model was $66 \%$; the sensitivity was $70 \%$.

\section{DISCUSSION}

\subsection{Main Findings}

There are many studies which investigate myocardial infarction. The unique characteristic of this study is its perspective on participants with a history of smoking. Our initial hypothesis was that the participants in our study who had experienced at least one of the critical life events measured would be at greater risk of myocardial infarction. Contrary to the hypothesis, however, the study found a negative association between the most frequent critical life events and myocardial infarction, meaning that the experience of at least one critical life event related to a personal accident, severe illness or changes at work tended to decrease the risk of myocardial infarction rather than increase it. The life events we investigated are drastic changes or problems which were typical for middle-aged to older adults [7], like most participants in CoSmoS. The patients were recruited in three different control clinics, so the issue of selection bias was to a large extent avoided. Owing to the study's limited sample size, however, these results should be interpreted with caution.

The study by Kornerup et al. [12] and the study by Hollis's team of researchers [13] yielded results similar to ours, finding no positive correlation between life events and cardiovascular outcomes. Critical life events generally have huge impacts on a person's life. On average, our sample, partially consisting of seriously-ill patients, had experienced few of the types of critical life events included in the study. At the same time, the surveyed patients also seemed to possess appropriate resources for compensating for these events, thereby protecting themselves from further damage to their health. This could imply that the healthy patients (without myocardial infarction and without critical life events) were better able to process critical life situations and, as a result, avoid any consequential illness or disease [20].

In a recently published article, Carey [21] reports on a study which found that experience of certain life events could have a positive effect on health, as in the saying "What doesn't kill you, makes you stronger". It is possible that these people develop adequate resources and strength until a critical life event. Participants who do not suffer from myocardial infarction directly after the critical life event seem to be robust. The other possibility is that people who had a critical life event, and developed a myocardial infarction soon afterwards, died and were not therefore included in this study sample. Equally, a critical life event itself could be responsible for the death of a person. It may also be that different rules apply to people with a history of smoking.

Other variables such as physical activity, age and gender seemed to have a greater effect on the onset of myocardial infarction.

Results from our study sample indicate that physical activity has a protective effect - albeit limited - on myocardial infarction. Similarly, Williams [22] reported physical activity to have little effect on decreases in the risk of myocardial infarction. Apart from the positive impact on quality of life [23], physical activity can have on perceived health, it has also long been proven to have a positive impact on the physiology of the heart [24]. The fact of growing older also plays an important role in the development of myocardial infarction. Our results suggesting that men are at higher risk of myocardial infarcttion correspond with data from the WHO which indicated a higher prevalence of myocardial infarction among men [3]. This does not mean, however, that myocardial infarction is an exclusively male condition [25].

Our findings underscore the discussion above in that the occurrence of critical life events may not play a special role in the onset of illnesses. Among the patients in our study, other causes seemed to contribute more to the onset of myocardial infarction.

\subsection{Limitations of the Study}

Owing to the retrospective nature of the study and the pathophysiological process of myocardial infarction (potentially extending over many years), it could not be ascertained whether stressful life events alone are related to the onset of illness or its clinical manifestations [11].

Unlike the other studies mentioned above, CoSmoS surveyed severely-ill participants. Face-to-face interviews therefore had to be conducted in hospital and were not anonymous [26]. Social desirability also seemed to play a major role in the response behaviour of the participants [27]. Furthermore, this retrospective survey was probably an underpowered substudy of a heterogeneous population. Owing to the sample size, the number of independent variables studied for their association with myocardial infarction had to be limited. An excess of parameters and associated overfitting of the data would have led to unstable regression coefficient estimates [28]. In this study the patients were usually surveyed immediately after they suffered a myocardial infarction. As a result, in addition to potential memory distortions in the patients' responses about critical life events they had experienced, cognitive distortions may have arisen owing to the trauma of having suffered from a life-threatening 
disease. In such boundary situations, the subjective assessments or personality dimensions being measured may be distorted, inevitably limiting an assessment of the association between personality and the risk of myocardial infarction [9].

As demonstrated by the numerous studies discussed above, the Social Readjustment Scale can be used in different ways. According to Weber [10], one limitation of the Social Readjustment Scale is that it does not include "non-events", such as unwanted pregnancy or the inability to conceive.

\subsection{Future Research}

Both the findings of previous studies and the findings of this study indicate a need for further investigations. Future research should include prospective studies with larger samples of smokers and ex-smokers from various professional fields. A larger sample of non-inpatients would allow for the inclusion and study of more critical life events. Prospective study designs could be used to determine the impact of these events on myocardial infarction and whether a combination of different life events together with sociodemographic data have any particularly "disastrous" effects. A study with a larger sample of participants with a history of smoking could investigate whether stressful life events are associated with smoking status [29]. There are many factors determining whether a critical life event will result in a crisis situation with an unfavourable outcome or possibly even lead to wisdom and personal growth [30]. Future research could place more emphasis on the intermediary process.

\subsection{Policy and Practice Implications}

Even though the results of this study indicate that the experience of critical life events reduces the risk of myocardial infarction, treatment teams should still inquire about events occurring in their patients' lives and take these into account during the course of treatment when necessary. Psychosocial dimensions should therefore also play an important role in myocardial infarction prevention and treatment alongside the necessary medical procedures [11]. Although in most cases the occurrence of critical life events cannot be prevented, it can be assumed that each person perceives and processes these relatively unwanted experiences differently. Results from the patients studied also suggested that physical activity is a reasonable complement to myocardial infarction prevention measures [31].

For the risk group "current smokers", smoking cessation should also be considered for inclusion in therapeutic regimens. As numerous other studies have shown, the risk of heart disease decreases shortly after quitting smoking [32].

\section{CONCLUSION}

The results of this study showed that sociodemographic data and physical activity have an effect on the development of myocardial infarction. Our unexpected study results showed that the experience of critical life events seemed to have a positive effect on health and could prevent myocardial infarction. Participants appear to show strength after the event and possess adequate resources to protect their health. Further prospective studies are needed to investigate the importance of psychosocial factors like critical life events.

\section{ACKNOWLEDGEMENTS}

This work was supported by the Helmholtz Association of German Research Centres [grant number VH-VI-143] and the Else KrönerFresenius-Stiftung [P25/09//A37/09]. Dr Melanie Neumann was supported by a grant from the Software AG Foundation, Germany. The authors would like to thank all patients for their active participation in this study as well as all of the cooperating clinics and institutes for their help in conducting the study. We are also grateful to Fawn Zarkov for her expert help with the English language.

\section{REFERENCES}

[1] Englert, H.S., Diehl, H.A., Greenlaw, R.L., Willich, S.N. and Aldana, S. (2007) The effect of a community-based coronary risk reduction: The Rockford CHIP. Preventive Medicine, 44, 513-519. doi:10.1016/j.ypmed.2007.01.014

[2] WHO (2008) The top 10 causes of death. http://www.who.int/mediacentre/factsheets/fs310/en/

[3] WHO (2006) Numbers and rates of registered deaths. http://apps.who.int/whosis/database/mort/table1_process. $\underline{\mathrm{cfm}}$

[4] Bullen, C. (2008) Impact of tobacco smoking and smoking cessation on cardiovascular risk and disease. Expert Review of Cardiovascular Therapy, 6, 883-895. doi:10.1586/14779072.6.6.883

[5] American Cancer Society, Inc., World Health Organization and International Union against Cancer (2003) Tobacco control country profiles, Atlanta. http://www.cancer.org/Healthy/InformationforHealthCare Professionals/tobacco-control-country-profiles

[6] Oliveira, A., Barros, H., Azevedo, A., Bastos, J. and Lopes, C. (2009) Impact of risk factors for non-fatal acute myocardial infarction. European Journal of Epidemiology, 24, 425-432. doi:10.1007/s10654-009-9352-9

[7] Schmitz, U., Rothermund, K. and Brandtstädter, J. (1999) Personality and life events: Predictive relationships. $\mathrm{Pa}$ dagogische Psychologie, 31, 147-156.

[8] Holmes, T. and Rahe, R. (1967) The social readjustment rating scale. Journal of Psychosomatic Research, 11, 213218. doi:10.1016/0022-3999(67)90010-4

[9] Geyer, S. (2003) The role of life-changing events in the 
onset and course of a heart attack. In: Slesina, W. and Werdan, K., Eds., Psychosocial Factors of Coronary Heart Disease, Schattauer, Stuttgart, 13-21.

[10] Weber, H. (2002) Stress measurement. In: Schwarzer, R., Jerusalem, M. and Weber, H., Eds., Health Psychology from A to Z. A Concise Dictionary, Hogrefe, Göttingen, 582-586.

[11] Rafanelli, C., Roncuzzi, R., Milaneschi, Y., Tomba, E., Colistro, M.C., Pancaldi, L.G., et al. (2005) Stressful life events, depression and demoralization as risk factors for acute coronary heart disease. Psychotherapy Psychosomatics, 74, 179-184. doi:10.1159/000084003

[12] Kornerup, H., Osler, M., Boysen, G., Barefoot, J., Schnohr, P. and Prescott, E. (2010) Major life events increase the risk of stroke but not of myocardial infarction: Results from the Copenhagen city heart study. European Journal of Cardiovascular Prevention \& Rehabilitation, 17, 113-118. doi:10.1097/HJR.0b013e3283359c18

[13] Hollis, J.F., Connett, J.E., Stevens, V.J. and Greenlick, M.R. (1990) Stressful life events, type A behavior, and the prediction of cardiovascular and total mortality over six years. Journal of Behavioral Medicine, 13, 263-279. doi:10.1007/BF00846834

[14] Guillemin, F., Krol, B., Briancon, A., Sanderman, R., Suurmeijer, T., Doeglas, D., et al. (1995) Stressful life events and disability in early rheumatoid arthritis. European Journal of Public Health, 5, 163-168. doi:10.1093/eurpub/5.3.163

[15] Jung, J., Neumann, M., Ernstmann, N., Wirtz, M., Staratschek-Jox, A., Wolf, J., et al. (2010) Validation of the "SmoCess-GP" instrument-A short patient questionnaire for assessing the smoking cessation activities of general practitioners: A cross-sectional study. BMC Family Practice, 11, 9. doi:10.1186/1471-2296-11-9

[16] Bodenmann, G., Schwerzmann, S. and Cina, A. (2000) Critical life events and daily life stress of patients with depression and depression in remission. Zeitschrift für Klinische Psychologie und Psychotherapie, 48, 1-17.

[17] Janjigian, Y.Y., McDonnell, K., Kris, M.G., Shen, R., Sima, C.S., Bach, P.B., et al. (2010) Pack-years of cigarette smoking as a prognostic factor in patients with stage IIIB/IV nonsmall cell lung cancer. Cancer, 116, 670-675. doi:10.1002/cncr.24813

[18] Onega, T., Goodrich, M., Dietrich, A. and Butterly, L. (2010) The influence of smoking, gender, and family history on colorectal adenomas. Journal of Cancer Epidemiology, 2010, Article ID: 509347. doi:10.1155/2010/509347

[19] Katz, M.H. (2006) Multivariable analysis: A practical guide for clinicians. Cambridge University Press, Cambridge. doi:10.1017/CBO9780511811692

[20] Seery, M.D., Holman, E.A. and Silver, R.C. (2010) What- ever does not kill us: Cumulative lifetime adversity, vulnerability, and resilience. Journal of Personality and Social Psychology, 99, 1025-1041. doi:10.1037/a0021344

[21] Carey, B. (2011) On road to recovery, past adversity provides a map. The New York Times, New York.

[22] Williams, P.T. (2010) Usefulness of cardiorespiratory fitness to predict coronary heart disease risk independent of physical activity. American Journal of Cardiology, 106, 210-215. doi:10.1016/j.amjcard.2010.03.017

[23] Pernambuco, C., Rodrigues, B., Bezerra, J., Carrielo, A., Fernandes, A., Vale, R. and Dantas, E. (2012) Quality of life, elderly and physical activity. Health, 4, 88-93. doi:10.4236/health.2012.42014

[24] Lampert, T. (2010) Smoking, physical inactivity, and obesity: Associations with social status. Deutsches Ärzteblatt, 107, 1-7.

[25] Löwel, H., Meisinger, C., Heier, M., Hörmann, A., Kuch, B., Gostomzyk, J., et al. (2002) Sex-specific trends of sudden cardiac death and acute myocardial infarction: Results of the population-based KORA/MONICA-Augsburg register 1985 to 1998. Deutsche Medizinische Wochenzeitschrift, 44, 2311-2316.

[26] Stein, L.A.R., Colby, S.M., O’Leary, T.A., Monti, P.M., Rohsenow, D.J., Spirito, A., et al. (2002) Response distortion in adolescents who smoke: A pilot study. Journal of Drug Education, 32, 271-286. doi:10.2190/GL7E-B8MV-P9NH-KCVV

[27] Schmidt, A., Neumann, M., Wirtz, M., Ernstmann, N., Staratschek-Jox, A., Stoelben, E., et al. (2010) The influence of occupational stress factors on nicotine dependence: A cross-sectional study. Tobacco Induced Disease, 8, 6. doi:10.1186/1617-9625-8-6

[28] Muche, R. (2008) Logistic regression: A useful tool in rehabilitation research. Rehabilitation, 47, 56-62. doi:10.1055/s-2007-992790

[29] McKee, S.A., Maciejewski, P.K., Falba, T. and Mazure, C.M. (2003) Sex differences in the effect of stressful life events on changes in smoking status. Addiction, 98, 847855. doi:10.1046/j.1360-0443.2003.00408.x

[30] Filipp, S.-H. (2002) Life events, critical. In: Schwarzer, R., Jerusalem, M. and Weber, H., Eds., Health Psychology from A to Z. A Concise Dictionary, Hogrefe, Göttingen, 345-348.

[31] National Institue for Health and Clinical Excellence (2010) NICE public health guidance 25: Prevention of cardiovascular disease at population level. http://guidance.nice.org.uk/PH25

[32] Edwards, R. (2004) ABC of smoking cessation: The problem of tobacco smoking. British Medical Journal, 328, 217-219. doi:10.1136/bmj.328.7433.217 